\title{
Transition from inspiral to plunge for a compact body in a circular equatorial orbit around a massive, spinning black hole
}

\author{
Amos Ori \\ Department of Physics, Technion-Israel Institute of Technology, Haifa, 32000, Israel \\ Kip S. Thorne \\ Theoretical Astrophysics, California Institute of Technology, Pasadena, California 91125
}

(Received 22 February 2000; published 28 November 2000)

\begin{abstract}
There are three regimes of gravitational-radiation-reaction-induced inspiral for a compact body with mass $\mu$, in a circular, equatorial orbit around a Kerr black hole with mass $M \gg \mu$ : (i) the adiabatic inspiral regime, in which the body gradually descends through a sequence of circular, geodesic orbits; (ii) a transition regime, near the innermost stable circular orbit (isco); (iii) the plunge regime, in which the body travels on a geodesic from slightly below the isco into the hole's horizon. This paper gives an analytic treatment of the transition regime and shows that, with some luck, gravitational waves from the transition might be measurable by the space-based LISA mission.
\end{abstract}

PACS number(s): 04.30.Db, 04.80.Nn, 97.60.Lf

\section{INTRODUCTION AND SUMMARY}

The space-based Laser Interferometer Space Antenna (LISA) [1], if it flies, is likely to detect and study the gravitational waves from white dwarfs, neutron stars and small black holes with masses $\mu \gtrsim 1 M_{\odot}$, spiraling into massive $\left(M \sim 10^{5}-10^{8} M_{\odot} \gg \mu\right)$ black holes in the nuclei of distant galaxies [2-4]. In preparation for these studies, it is necessary to understand, theoretically, the radiation-reactioninduced evolution of the inspiral orbits, and the gravitational wave forms that they emit.

Regardless of an orbit's shape and orientation, when $\mu$ $\ll M$ the orbital evolution can be divided into three regimes: (i) the adiabatic inspiral regime, in which the body gradually descends through a sequence of geodesic orbits with gradually changing "constants" of the motion $E=$ (energy), $L=$ (polar component of angular momentum), and $Q=$ (Carter constant); (ii) a transition regime, in which the character of the orbit gradually changes from inspiral to plunge. (iii) a plunge regime, in which the body plunges into the horizon along a geodesic with (nearly) unchanging $E, L$ and $Q$.

The plunge regime, being (essentially) ordinary geodesic motion, is well understood; and the adiabatic inspiral regime is the focus of extensive current research (see, e.g., [4-6]). By contrast, so far as we are aware, there have been no publications dealing with the transition regime.

We begin, in Sec. II, by summarizing some key, wellknown details of the inspiral and plunge regimes. Then in Sec. III A we present a qualitative picture of the transition from inspiral to plunge, based on the motion of a particle in a slowly changing effective potential (Fig. 1). With the aid of this qualitative picture, in Sec. III B we derive a nongeodesic equation of motion for the transition regime, and in Sec. III C we construct the solution to that equation of motion (Figs. 2 and 3). Then in Sec. IV, with the aid of our solution, we estimate the gravitational-wave signal strength from the transition regime and the signal-to-noise ratio that it would produce in LISA. We conclude that, with some luck,
LISA may be able to detect and study the transition waves. In Sec. V we make concluding remarks about the need for further research.

\section{ADIABATIC INSPIRAL AND PLUNGE}

Throughout this paper we use Boyer-Lindquist coordinates $(t, r, \theta, \phi)[7]$ for the massive hole's Kerr metric, and we use geometrized units, with $G=c=1$. The hole's mass is $M$ and the inspiraling body's mass is $\mu \equiv \eta M$. We use $M$ and $\mu$ to construct dimensionless versions (denoted by tildes) of many dimensionfull quantities; for example, $\tilde{r}=r / M$, and $\tilde{t}$ $=t / M$. The hole's dimensionless spin parameter is $a \equiv$ (spin angular momentum) $/ M^{2}$ (with $-1<a<+1$ ). The body moves around its circular, equatorial orbit in the $+\phi$ direction, so $a>0$ corresponds to an orbit that is prograde relative to the hole's spin, and $a<0$ to a retrograde orbit.

When the inspiraling body is not too close to the innermost stable circular orbit (isco), it moves on a circular geodesic orbit with dimensionless angular velocity [8]

$$
\widetilde{\Omega} \equiv M \Omega=\frac{d \phi}{d \tilde{t}}=\frac{1}{\widetilde{r}^{3 / 2}+a}
$$

(where $\phi$ is angle around the orbit) and with orbital energy [8]

$$
E=-\eta M \frac{1-2 / \tilde{r}+a / \tilde{r}^{3 / 2}}{\sqrt{1-3 / \tilde{r}+2 a / \tilde{r}^{3 / 2}}} .
$$

As it moves, the body radiates energy into gravitational waves at a rate given by [4] 
TABLE I. Dimensionless parameters characterizing the isco and the transition regime of inspiral. The values of $\dot{\mathcal{E}}$ are from numerical solutions of the Teukolsky equation by L. S. Finn (first line of Table II of Ref. [4]).

\begin{tabular}{lcccccccc}
\hline \hline \multicolumn{1}{c}{$a$} & $\tilde{r}_{\text {isco }}$ & $\widetilde{\Omega}_{\text {isco }}$ & \multicolumn{1}{c}{$\alpha$} & \multicolumn{1}{c}{$\alpha$} & \multicolumn{1}{c}{$\beta$} & \multicolumn{1}{c}{$\kappa$} & $R_{o}$ & $\tau_{o}$ \\
\hline-0.99 & 8.972 & 0.03863 & 1.240 & 0.0001543 & 0.006626 & 0.005013 & 3.129 & 45.50 \\
-0.9 & 8.717 & 0.04026 & 1.233 & 0.0001732 & 0.007070 & 0.005527 & 3.117 & 43.04 \\
-0.5 & 7.555 & 0.04935 & 1.197 & 0.0003070 & 0.009730 & 0.008966 & 3.048 & 32.69 \\
0 & 6.000 & 0.06804 & 1.143 & 0.0007716 & 0.01604 & 0.01955 & 2.925 & 21.05 \\
0.2 & 5.329 & 0.07998 & 1.114 & 0.001240 & 0.02057 & 0.02914 & 2.852 & 16.82 \\
0.5 & 4.233 & 0.1086 & 1.053 & 0.003115 & 0.03270 & 0.06291 & 2.687 & 10.93 \\
0.8 & 2.907 & 0.1737 & 0.9144 & 0.01401 & 0.06446 & 0.2123 & 2.326 & 5.539 \\
0.9 & 2.321 & 0.2254 & 0.7895 & 0.03447 & 0.09039 & 0.4214 & 2.041 & 3.770 \\
0.99 & 1.454 & 0.3644 & 0.4148 & 0.2234 & 0.1289 & 1.531 & 1.284 & 1.867 \\
0.999 & 1.182 & 0.4379 & 0.2022 & 0.5127 & 0.09568 & 2.594 & 0.8551 & 1.510 \\
\hline \hline
\end{tabular}

$$
\dot{E}_{\mathrm{GW}}=-\dot{E}=\frac{32}{5} \eta^{2} \widetilde{\Omega}^{10 / 3} \dot{\mathcal{E}},
$$

where $\dot{\mathcal{E}}$ is a general relativistic correction to the Newtonian, quadrupole-moment formula (Table II of Ref. [4]). This energy loss causes the orbit to shrink adiabatically at a rate given by

$$
\frac{d r}{d t}=\frac{-\dot{E}_{\mathrm{GW}}}{d E / d r}
$$

The inspiral continues adiabatically until the body nears the isco, which is at the dimensionless radius $\tilde{r}_{\text {isco }}=r_{\text {isco }} / M$ given by [8]

$$
\begin{aligned}
\tilde{r}_{\mathrm{isco}} & =3+Z_{2}-\operatorname{sgn}(a)\left[\left(3-Z_{1}\right)\left(3+Z_{1}+2 Z_{2}\right)\right]^{1 / 2}, \\
Z_{1} & \equiv 1+\left(1-a^{2}\right)^{1 / 3}\left[(1+a)^{1 / 3}+(1-a)^{1 / 3}\right], \\
Z_{2} & \equiv\left(3 a^{2}+Z_{1}^{2}\right)^{1 / 2} ;
\end{aligned}
$$

cf. Table I. The circular geodesic orbit at the isco has dimensionless angular velocity (Table I), energy, and angular momentum given by $[8,7]$

$$
\begin{aligned}
& \widetilde{\Omega}_{\mathrm{isco}} \equiv M \Omega=\frac{1}{\widetilde{r}_{\mathrm{isco}} 3 / 2+a}, \\
& \widetilde{E}_{\mathrm{isco}} \equiv \frac{E_{\mathrm{isco}}}{\mu}=\frac{E_{\mathrm{isco}}}{\eta M}=\frac{1-2 / \tilde{r}_{\mathrm{isco}}+a / \tilde{r}_{\mathrm{isco}} 3 / 2}{\sqrt{1-3 / \tilde{r}_{\mathrm{isco}}+2 a / \tilde{r}_{\mathrm{isco}} 3 / 2}}
\end{aligned}
$$

$$
\widetilde{L}_{\text {isco }} \equiv \frac{L_{\text {isco }}}{\mu M}=\frac{L_{\text {isco }}}{\eta M^{2}}=\frac{2}{\sqrt{3 \tilde{r}_{\text {isco }}}}\left(3 \sqrt{\tilde{r}_{\text {isco }}}-2 a\right) .
$$

As the body nears the isco, its inspiral gradually ceases to be adiabatic and it enters the transition regime (Sec. III). Radiation reaction (as controlled by $\dot{E}_{\mathrm{GW}}$ ) continues to drive the orbital evolution throughout the transition regime, but gradually becomes unimportant as the transition ends and pure plunge takes over.

The plunge is described to high accuracy by reaction-free geodesic motion; Eqs. (33.32) of Ref. [7]. Up to fractional corrections of order $\eta^{4 / 5}$, the orbital energy and angular momentum of the plunging body are equal to $E_{\text {isco }}$ and $L_{\text {isco }}$ throughout the plunge [cf. Eq. (3.26) below].

\section{THE TRANSITION FROM ADIABATIC INSPIRAL TO PLUNGE}

\section{A. Qualitative explanation of transition}

As the body nears its innermost stable circular orbit, $r$ $=r_{\text {isco }}$, the adiabatic approximation begins to break down. This breakdown can be understood in terms of the effective potential, which governs geodesic radial motion via the equation

$$
\left(\frac{d \tilde{r}}{d \tilde{\tau}}\right)^{2}=\left(\frac{d r}{d \tau}\right)^{2}=\widetilde{E}^{2}-V(\tilde{r}, \widetilde{E}, \widetilde{L}),
$$

where $\widetilde{E} \equiv E / \mu=E /(\eta M), \widetilde{L} \equiv L /(\mu M)=L /\left(\eta M^{2}\right)$, and $\tilde{\tau}$ $\equiv \tau / M$ are the body's dimensionless energy, angular momentum, and proper time. The explicit form of the effective potential can be inferred from Eqs. (33.32) and (33.33) of Misner, Thorne and Wheeler (MTW) [7]:

$$
\begin{aligned}
V(\tilde{r}, \widetilde{E}, \widetilde{L})= & \widetilde{E}^{2}-\frac{1}{\widetilde{r}^{4}}\left(\left[\widetilde{E}\left(\widetilde{r}^{2}+a^{2}\right)-\widetilde{L} a\right]^{2}\right. \\
& \left.-\left(\widetilde{r}^{2}-2 \widetilde{r}+a^{2}\right)\left[\widetilde{r}^{2}+(\widetilde{L}-\widetilde{E} a)^{2}\right]\right) .
\end{aligned}
$$


For a Schwarzschild black hole, this reduces to

$$
V(\tilde{r}, \widetilde{E}, \widetilde{L})=\left(1-\frac{2}{\tilde{r}}\right)\left(1+\frac{\widetilde{L}^{2}}{\widetilde{r}^{2}}\right) \quad \text { for } a=0
$$

[cf. Eq. (25.16) of MTW [7]].

Throughout the inspiral and transition regimes, the body moves along a nearly circular orbit; its change of radius during each circuit around the hole is $\Delta r \ll r$. (Only after the body is well into its final plunge toward the hole does $\Delta r$ become comparable to $r$.) This near-circular motion guarantees that the ratio of the energy radiated to angular momentum radiated is equal to the body's orbital angular velocity [9]:

$$
\frac{d \tilde{E}}{d \tilde{\tau}}=\widetilde{\Omega} \frac{d \tilde{L}}{d \tilde{\tau}}
$$

Correspondingly, in and near the transition regime, which occupies a narrow range of radii around $\tilde{r}_{\text {isco }}$, the body's energy and angular momentum are related by ${ }^{1}$

$$
\widetilde{E}=\widetilde{E}_{\text {isco }}+\widetilde{\Omega}_{\text {isco }} \xi, \quad \widetilde{L}=\widetilde{L}_{\text {isco }}+\xi
$$

By combining Eqs. (3.5) and (3.2), we can regard the body's effective potential as a function of $\tilde{r}$ and the difference $\xi$ $\equiv \widetilde{L}-\widetilde{L}_{\text {isco }}$ of its orbital angular momentum from that of the isco.

Figure 1 shows $V(\tilde{r}, \xi)$ for a sequence of angular momenta $\xi_{1}, \ldots, \xi_{5}$ around $\xi=0$. As $\xi$ decreases to $\xi=0$, the minimum of the potential flattens out and disappears; and just when it is disappearing, the minimum's radius $r_{\min }$ is moving inward at an infinite rate: $d r_{\min } / d \xi \rightarrow \infty$ as $\xi \rightarrow 0$.

In the adiabatic regime of large $\xi$, the body sits always at the minimum of the effective potential. Its orbit is a slowly shrinking circle, guided inward by the motion of the minimum. As $\xi$ nears zero and the minimum's inward speed grows large, the body's inertia prevents it from continuing to follow the minimum. The body begins to lag behind, as depicted at $\xi=\xi_{2}$ in Fig. 1. This lag invalidates the adiabatic inspiral analysis of Sec. II and initiates the transition regime.

\footnotetext{
${ }^{1}$ In reality, finite-mass-ratio effects, including those discussed in the paragraph preceding Eq. (3.11) below, will alter these energyangular-momentum relations by amounts that scale as the first and higher powers of $\eta$. For example, in going from Eq. (3.4) to (3.5), there can be an integration constant $\delta \widetilde{E}$ (which scales as $\eta$ or some higher power) so $\widetilde{E}=\widetilde{E}_{\text {isco }}+\delta \widetilde{E}+\widetilde{\Omega}_{\text {isco }} \xi$. In the presence of such effects, we redefine $\tilde{r}_{\text {isco }}, \widetilde{E}_{\text {isco }}$, and $\widetilde{L}_{\text {isco }}$ to be the values of these parameters at which the $\eta$-corrected $V(\tilde{r}, \widetilde{E}, \widetilde{L})$ has a flat inflection point, as in Fig. 1, and $\widetilde{\Omega}_{\text {isco }}$ to be the orbital angular velocity at this $\tilde{r}_{\text {isco }}$. Then Eqs. (3.5) remain valid even for finite mass ratio $\eta$.
}

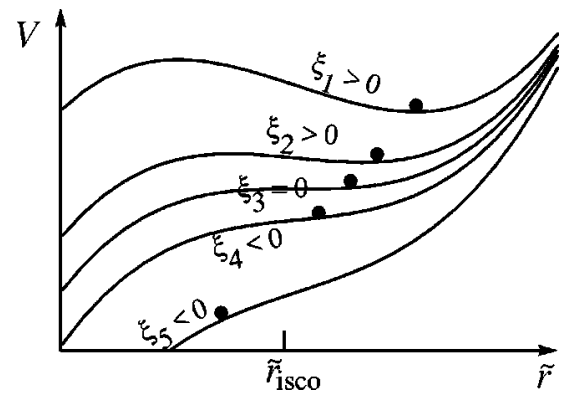

FIG. 1. The gradually changing effective potential $V(\tilde{r}, \xi)$ for radial geodesic motion. Each curve is for a particular value of $\xi$ $\equiv \widetilde{L}-\widetilde{L}_{\text {isco }}$. As $\xi$ decreases due to radiation reaction, the body, depicted by the large dot, at first remains at the minimum of the effective potential ( $\xi_{1}$; " adiabatic regime"). However, as $\xi$ nears zero (at $\xi \simeq \xi_{2}$ ), the body cannot keep up with the rapid inward motion of the minimum; it lags behind in a manner described by the transition-regime analysis of Sec. III. At $\xi \simeq \xi_{5}$ the effective potential has become so steep that radiation reaction is no longer important, the transition regime ends, and the body plunges toward the black hole with nearly constant energy and angular momentum.

As $\xi$ continues to decrease, there comes a point (near $\xi_{5}$ in Fig. 1) at which the effective potential has become so steep that its inward force on the body dominates strongly over radiation reaction. There the transition regime ends, and the body begins to plunge inward rapidly on a nearly geodesic orbit with nearly constant $\widetilde{E}$ and $\widetilde{L}$. The objectives of the following subsections are to derive a set of equations describing the transition regime (Sec. III B), and show how the transition matches smoothly onto the adiabatic regime at large positive $\xi$ and to the plunge regime at large negative $\xi$ (Sec. III C).

\section{B. Equation of motion for transition regime}

Throughout the transition regime, because the body moves on a nearly circular orbit with radius close to $r_{\text {isco }}$, and because the body's small mass $\mu \equiv \eta M \ll M$ keeps its radiation reaction weak, its angular velocity remains very close to $\Omega_{\text {isco }}$,

$$
\frac{d \phi}{d \tilde{t}} \equiv \widetilde{\Omega} \simeq \widetilde{\Omega}_{\mathrm{isco}},
$$

and its proper time ticks at very nearly the standard isco rate

$$
\frac{d \tilde{\tau}}{d \tilde{t}} \simeq\left(\frac{d \tilde{\tau}}{d \tilde{t}}\right)_{\mathrm{isco}}=\frac{\sqrt{1-3 / \tilde{r}_{\mathrm{isco}}+2 a / \tilde{r}_{\mathrm{isco}} 3 / 2}}{1+a / \tilde{r}_{\mathrm{isco}} 3 / 2}
$$

cf. Eq. (5.4.5a) of [10].

This nearly circular motion at $\tilde{r} \simeq \tilde{r}_{\text {isco }}$ produces gravitational waves which carry off angular momentum and energy at very nearly the same rate as they would for circular geo-

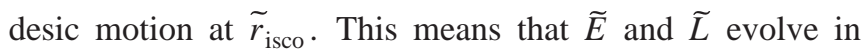
accord with Eqs. (3.5), where 


$$
\frac{d \xi}{d \tilde{\tau}}=-\kappa \eta
$$

and

$$
\kappa=\frac{32}{5} \widetilde{\Omega}_{\mathrm{isco}} 7 / 3 \frac{1+a / \tilde{r}_{\mathrm{isco}} 3 / 2}{\sqrt{1-3 / \tilde{r}_{\mathrm{isco}}+2 a / \tilde{r}_{\mathrm{isco}} 3 / 2}} \dot{\mathcal{E}}_{\mathrm{isco}} ;
$$

cf. Eqs. (2.3), (3.5), (3.7), and Table I. It is the smallness of $\eta \equiv \mu / M$ (e.g., $\eta=10^{-5}$ for the realistic case of a $10 M_{\odot}$ black hole spiraling into the $10^{6} M_{\odot}$ black hole) that makes the angular momentum $\xi$ evolve very slowly and keeps the body in a nearly circular orbit throughout the transition regime [cf. the factors of $\eta$ that appear in Eqs. (3.8) and (3.20) — which with Eqs. (3.11) and (3.22) imply $d \tilde{r} / d \tilde{\tau}$ $\left.\propto \eta^{3 / 5}\right]$.

In the transition regime, the body's radial motion is described by the geodesic equation of motion with a radial self force per unit mass ${ }^{2} \eta \widetilde{F}_{\text {self }}$ inserted on the right-hand side:

$$
\frac{d^{2} \tilde{r}}{d \tilde{\tau}^{2}}=-\frac{1}{2} \frac{\partial V(\tilde{r}, \xi)}{\partial \tilde{r}}+\eta \widetilde{F}_{\text {self }}
$$

(We write it as $\eta \widetilde{F}_{\text {self }}$ because its magnitude is proportional to $\eta=\mu / M$.)

The radial self-force $\eta \widetilde{F}_{\text {self }}$ is nondissipative (since it has hardly any radial velocity with which to couple). This contrasts with the $\phi$-directed radiation-reaction force, which couples to the orbital angular velocity to produce a shrinkage of the body's angular momentum [Eq. (3.8)] and a corresponding decrease of its energy, $d \widetilde{E} / d \tilde{\tau}=\widetilde{\Omega} d \xi / d \tilde{\tau}$. Because the radial force is nondissipative, it is of little importance. It can be absorbed into the nondissipative effective potential term $-\frac{1}{2} \partial V / \partial \tilde{r}$ in the equation of motion. Doing so will not change the general character of the effective potential, as depicted in Fig. 1; it will merely change, by fractional amounts proportional to $\eta$, the various parameters that characterize the effective potential: the location $\tilde{r}_{\text {isco }}$ of the innermost stable circular orbit (at which the $\xi=0$ effective potential curve has its inflection point), the values at the isco of the orbital energy and angular momentum $\widetilde{E}_{\text {isco }}$ and $\widetilde{L}_{\text {isco }}$, and the constant $\alpha$ defined below. There will be other $\mathrm{O}(\eta)$ changes in $\widetilde{r}_{\text {isco }}, \widetilde{E}_{\text {isco }}, \widetilde{L}_{\text {isco }}$ and $\alpha$ caused by the body's own perturbation of the hole's spacetime geometry [11-13]. In

\footnotetext{
${ }^{2}$ This radial self-force, like the radiation reaction force that drives the inspiral, is produced by interaction of the body with its own gravitational field - that field having been influenced by the black hole's spacetime geometry; see, e.g., Ref. [6]. The contravariant radial component of the self-force, with dimensionality restored using $\quad r=M \tilde{r}$ and $\tau=M \tilde{\tau}, \quad$ is $\quad\left(d p^{r} / d \tau\right)_{\text {self }}=\left(\mu d^{2} r / d \tau^{2}\right)_{\text {self }}$ $=(\mu / M)\left(d^{2} \tilde{r} / d \tilde{\tau}^{2}\right)_{\text {self }}=\eta^{2} \widetilde{F}_{\text {self }}$.
}

this paper, we shall ignore all such changes, and correspondingly we shall neglect the radial self-force $\eta \widetilde{F}_{\text {self }}$.

We shall describe the body's location in the transition regime by

$$
R \equiv \tilde{r}-\tilde{r}_{\text {isco }}
$$

Throughout the transition regime both $R$ and $\xi$ are small, and correspondingly the effective potential can be expanded in powers of $R$ and $\xi$. Up through cubic terms in $R$ and linear terms in $\xi$ (the order needed for our analysis), the effective potential takes the form

$$
V(R, \xi)=\frac{2 \alpha}{3} R^{3}-2 \beta R \xi+\text { const },
$$

where $\alpha$ and $\beta$ are positive constants that we shall evaluate below. Note that for $\xi=0$, this is a simple cubic potential with inflection point at $R=0$, i.e., at $\tilde{r}=\widetilde{r}_{\text {isco }}$; and note that for $\xi>0$, it acquires a maximum and a minimum, while for $\xi<0$ it is monotonic; cf. Fig. 1. By inserting Eq. (3.12) into Eq. (3.10), setting $\tilde{r}=\tilde{r}_{\text {isco }}+R$, and neglecting the radial selfforce or absorbing it into $\tilde{r}_{\text {isco }}, \alpha$ and $\beta$ as described above, we obtain the following radial equation of motion:

$$
\frac{d^{2} R}{d \widetilde{\tau}^{2}}=-\alpha R^{2}+\beta \xi
$$

By then setting $\tilde{\tau} \equiv 0$ at the moment when $\xi=0$ and using Eq. (3.8) for the rate of change of $\xi$, so

$$
\xi=-\eta \kappa \tilde{\tau}
$$

we bring our equation of motion into the form

$$
\frac{d^{2} R}{d \tilde{\tau}^{2}}=-\alpha R^{2}-\eta \beta \kappa \tilde{\tau}
$$

We shall explore the consequences of this equation of motion in the next subsection, but first we shall deduce the values of $\alpha$ and $\beta$.

The constants $\alpha$ and $\beta$ can be evaluated from the following relations, which follow directly from Eqs. (3.12), (3.11) and (3.5):

$\alpha=\frac{1}{4}\left(\frac{\partial^{3} V(\tilde{r}, \widetilde{E}, \widetilde{L})}{\partial \widetilde{r}^{3}}\right)_{\text {isco }}$,

$\beta=-\frac{1}{2}\left(\frac{\partial^{2} V(\tilde{r}, \widetilde{E}, \widetilde{L})}{\partial \widetilde{L} \partial \tilde{r}}+\widetilde{\Omega} \frac{\partial^{2} V(\tilde{r}, \widetilde{E}, \widetilde{L})}{\partial \widetilde{E} \partial \tilde{r}}\right)_{\text {isco }}$

By inserting expression (3.2) into these relations, we obtain $\alpha$ and $\beta$ in the limit $\eta \equiv \mu / M \rightarrow 0$ : 


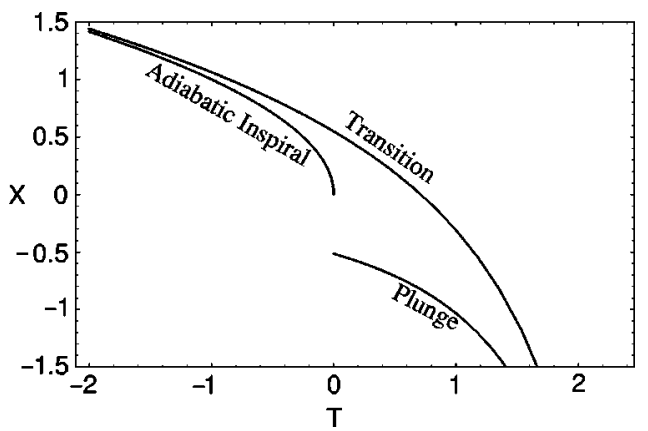

FIG. 2. Dimensionless orbital radius $X$ as a function of dimensionless proper time $T$ for an orbit near the isco. Adiabatic Inspiral: The analytic solution (3.23) for adiabatic inspiral outside the isco. Transition: The numerical solution to the dimensionless equation of motion (3.22) for the transition regime in the vicinity of the isco. Plunge: The analytic solution (3.25) for the orbital plunge inside the isco.

$$
\begin{aligned}
\alpha & =\frac{3}{\tilde{r}_{\text {isco }}^{6}}\left(\widetilde{r}^{2}+2\left[a^{2}\left(\widetilde{E}^{2}-1\right)-\widetilde{L}^{2}\right] \tilde{r}+10(\widetilde{L}-a \widetilde{E})^{2}\right)_{\text {isco }} \\
& =\frac{1}{1296} \text { for } a=0, \\
\beta & =\frac{2}{\widetilde{r}_{\text {isco }}^{4}}\left(\left(\widetilde{L}-a^{2} \widetilde{E} \widetilde{\Omega}\right) \tilde{r}-3(\widetilde{L}-a \widetilde{E})(1-a \widetilde{\Omega})\right)_{\text {isco }} \\
& =\frac{1}{36 \sqrt{3}} \quad \text { for } a=0 .
\end{aligned}
$$

Here $\widetilde{r}_{\text {isco }}$ and $\widetilde{\Omega}_{\text {isco }}$ are given by Eqs. (2.5) and (2.6); and $\widetilde{L}_{\text {isco }}$ and $\widetilde{E}_{\text {isco }}$ are expressed in terms of $\widetilde{r}_{\text {isco }}$ by Eqs. (2.7) and (2.8). Numerical values of $\alpha$ and $\beta$, computed from these equations, are tabulated in Table I.

\section{Solution for motion in the transition regime}

The equation of motion in the transition regime, Eq. (3.15), can be converted into dimensionless form by setting

$$
R=\eta^{2 / 5} R_{o} X, \quad \tilde{\tau}=\eta^{-1 / 5} \tau_{o} T,
$$

where

$$
R_{o}=(\beta \kappa)^{2 / 5} \alpha^{-3 / 5}, \quad \tau_{o}=(\alpha \beta \kappa)^{-1 / 5}
$$

cf. Table I. The resulting dimensionless equation of motion is

$$
\frac{d^{2} X}{d T^{2}}=-X^{2}-T
$$

We seek the unique solution of this differential equation which, at early times $T \ll-1$, joins smoothly onto the adiabatic inspiral solution of Sec. II. In that adiabatic inspiral, the orbit is the circle at the minimum of the effective potential of

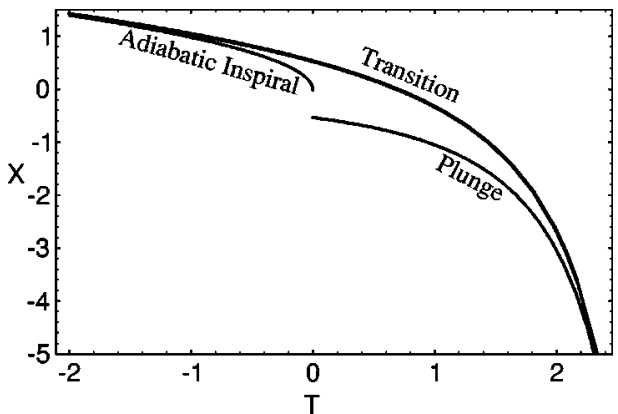

FIG. 3. Same as Fig. 2, but drawn on a different scale.

Fig. 1 and Eq. (3.12), i.e., the circle at $R=\sqrt{\beta \xi / \alpha}$ $=\sqrt{-\beta \kappa \eta \tau / \alpha}$, which translates into

$$
X=\sqrt{-T} \text { for adiabatic inspiral near the isco. }
$$

We have not been able to find an analytic formula for the solution to the equation of motion (3.22) that joins smoothly onto this adiabatic solution, but it is easy to construct the unique solution numerically. It is plotted in Figs. 2 and 3, along with the adiabatic inspiral solution (3.23) and the plunge solution [Eq. (3.25) below].

The transition solution is well approximated by adiabatic inspiral at times $T<-1$, but at $T>-1$ it deviates from adiabatic inspiral and evolves smoothly into a plunge. The solution diverges $(X \rightarrow-\infty)$ at a finite time $T=T_{\text {plunge }} \simeq 3.412 .^{3}$

In the plunge regime, radiation reaction is unimportant; i.e., the orbit evolves inward with (very nearly) constant orbital angular momentum $\widetilde{L} \simeq \widetilde{L}_{\text {final }}$ and energy $\widetilde{E} \simeq \widetilde{E}_{\text {final }}$ (which we evaluate below); i.e., the orbit is well approximated by geodesic free fall. In the dimensionless equation of motion (3.22), the free-fall approximation translates into neglecting the last term, $T$, so $d^{2} X / d T^{2}=-X^{2}$, which has the analytic first integral

$$
d X / d T=-\sqrt{\text { const }-\frac{2}{3} X^{3}} .
$$

For large $|X|$, the constant can be neglected and we obtain the analytic solution

\footnotetext{
${ }^{3}$ The divergence of $X$ at $T=T_{\text {plunge }}$ does not imply a divergence of $r$ or any other physical quantity. Rather, it marks the breakdown of the transition approximation at very large values of $|X|,|X|$ $\gtrsim X_{\text {break }} \sim \eta^{-2 / 5}$; [cf. Eq. (3.20)]. More specifically, when $-X$ $\geq X_{\text {break }}$, higher-order terms in the Taylor expansion (3.12) become important and stop the divergence. Well before this (in fact, throughout the range $1 \ll-X \ll X_{\text {break }}$ ) both the transition approximation and the free-fall approximation $\left(\widetilde{E}=\widetilde{E}_{\text {final }}=\right.$ constant, $\widetilde{L}$ $=\widetilde{L}_{\text {final }}=$ const ) are valid, so these two approximations can be matched in this regime to obtain a solution valid all the way down to the horizon. The same type of breakdown also occurs at the other asymptotic limit $+X \gtrsim X_{\text {break }}$ : The transition regime's adiabaticinspiral equation (3.23) breaks down and must be replaced, via matching at $1 \ll X \ll X_{\text {break }}$, by the exact Kerr metric's adiabatic inspiral formulas [4].
} 


$$
X=\frac{-6}{\left(T_{\text {plunge }}-T\right)^{2}} \quad \text { for plunge near the isco, }
$$

which is plotted in Figs. 2 and 3.

Combining Eqs. (3.5), (3.14), and (3.20), one finds that throughout the transition regime, the energy and angularmomentum deficits (i.e., the deviations of $\widetilde{E}$ and $\widetilde{L}$ from their isco values) scale as $\eta^{4 / 5}$. In particular, the final deficits in the plunge stage are given by

$$
\begin{aligned}
& \widetilde{L}_{\text {final }}-\widetilde{L}_{\text {isco }}=-\left(\kappa \tau_{0} T_{\text {plunge }}\right) \eta^{4 / 5}, \\
& \widetilde{E}_{\text {final }}-\widetilde{E}_{\text {isco }}=-\widetilde{\Omega}_{\text {isco }}\left(\kappa \tau_{0} T_{\text {plunge }}\right) \eta^{4 / 5},
\end{aligned}
$$

where, as was noted above,

$$
T_{\text {plunge }}=3.412 \text {. }
$$

\section{GRAVITATIONAL WAVES FROM TRANSITION REGIME, AND THEIR OBSERVABILITY}

The gravitational waves emitted in the transition regime are all near the orbital frequency $2 \pi \Omega_{\text {isco }}$ and its harmonics. The strongest waves are at the second harmonic (twice the orbital frequency):

$$
f \simeq 2 \frac{\Omega_{\text {isco }}}{2 \pi}=\frac{\widetilde{\Omega}_{\text {isco }}}{\pi M} .
$$

We shall compute their properties.

The transition waves last for a proper time $\Delta \tau=M \Delta \tilde{\tau}$ $=M \eta^{-1 / 5} \tilde{\tau}_{o} \Delta T$, during which the body spirals inward through a radial distance $\Delta r=M \Delta R=M \eta^{2 / 5} R_{o} \Delta X$, where $\Delta T$ covers the range $T \simeq-1$ to $\simeq 2.3$ and $\Delta X$ covers the range $X \simeq 1$ to $X \simeq-5$ (Fig. 3 ); i.e.,

$$
\Delta T=3.3, \quad \Delta X=6 .
$$

Correspondingly, neglecting any cosmological redshift, the duration of the transition waves as seen at Earth is

$$
\Delta t=\frac{M}{(d \tilde{\tau} / d \tilde{t})_{\text {isco }}} \eta^{-1 / 5} \tilde{\tau}_{o} \Delta T,
$$

and their frequency band is $\Delta f=(1 / \pi M)(d \widetilde{\Omega} / d \tilde{r})_{\text {isco }} \Delta \tilde{r}$, which, using the above expression for $\Delta r$ and Eq. (2.1) for $\widetilde{\Omega}(\tilde{r})$, gives

$$
\Delta f=\frac{3}{2 \pi M} \widetilde{\Omega}_{\mathrm{isco}}^{2} \sqrt{\widetilde{r}_{\mathrm{isco}}} \eta^{2 / 5} R_{o} \Delta X
$$

The total number of cycles of these transition waves is

$$
N_{\mathrm{cyc}}=f \Delta t=\frac{\widetilde{\Omega}_{\mathrm{isco}} \tilde{\tau}_{o}}{\pi(d \tilde{\tau} / d \tilde{t})_{\mathrm{isco}}} \eta^{-1 / 5} \Delta T .
$$

These second-harmonic waves arriving at Earth have the form $h_{+}=h_{+\mathrm{amp}} \cos \left(2 \pi \int f d t+\varphi_{+}\right), \quad h_{\times}=h_{\times \mathrm{amp}} \cos \left(2 \pi \int f d t\right.$ $\left.+\varphi_{\times}\right)$, where $\varphi_{+}$and $\varphi_{\times}$are constant phases. The amplitudes $h_{+ \text {amp }}$ and $h_{\times \text {amp }}$ depend on the source's orientation. When one squares and adds these amplitudes and then averages over the sky (" $\langle\ldots\rangle$ '), one obtains an rms amplitude:

$$
h_{\mathrm{amp}}^{\mathrm{rms}}=\left\langle h_{+\mathrm{amp}}^{2}+h_{\times \mathrm{amp}}^{2}\right\rangle^{1 / 2},
$$

which is related to the power being radiated into the second harmonic by $\dot{E}_{2}=4 \pi D^{2}\left(h_{\mathrm{amp}}^{\mathrm{rms}}\right)^{2}(2 \pi f)^{2} /(32 \pi)$; cf. Eq. (35.27) of MTW [7]. Here $D$ is the distance to the source. Equating this to the radiated power $\dot{E}_{2}=(32 / 5) \eta^{2} \widetilde{\Omega}_{\text {isco }}^{10 / 3} \dot{\mathcal{E}}_{\infty, 2}$ [4], where $\dot{\mathcal{E}}_{\infty, 2}$ is a relativistic correction factor listed on the first line of Table IV of [4], we obtain the following expression for the waves' rms amplitude

$$
h_{\mathrm{amp}}^{\mathrm{rms}}=\frac{8}{\sqrt{5}} \frac{M \eta}{D} \widetilde{\Omega}_{\mathrm{isco}}^{2 / 3} \sqrt{\dot{\mathcal{E}}_{\infty, 2}} .
$$

The signal to noise ratio $S / N$ that these waves produce in LISA depends on the orientations of LISA and the source relative to the line of sight between them. When one squares $S / N$ and averages over both orientations, then takes the square root, one obtains [14]

$$
\left(\frac{S}{N}\right)_{\mathrm{rms}}=\frac{h_{\mathrm{amp}}^{\mathrm{rms}}}{\sqrt{5 S_{h}(f) / \Delta t}} .
$$

Here $5 S_{h}(f)$ is the spectral density of LISA's strain noise inverse-averaged over the $\mathrm{sky}^{4}$ and $1 / \Delta t$ is the band width associated with the waves' duration $\Delta t$.

The noise spectral density $S_{h}(f)$ for the current straw-man design of LISA has been computed by the LISA Mission Definition Team [15]. An analytic fit to this $S_{h}(f)$, after averaging over some small-amplitude oscillations that occur at $f>0.01 \mathrm{~Hz}$, is the following:

$$
\begin{aligned}
S_{h}(f)= & {\left[\left(4.6 \times 10^{-21}\right)^{2}+\left(3.5 \times 10^{-26}\right)^{2}\left(\frac{1 \mathrm{~Hz}}{f}\right)^{4}\right.} \\
& \left.+\left(3.5 \times 10^{-19}\right)^{2}\left(\frac{f}{1 \mathrm{~Hz}}\right)^{2}\right] \mathrm{Hz}^{-1} .
\end{aligned}
$$

The rate for $\mu \sim 10 M_{\odot}$ black holes to spiral into $M$ $\sim 10^{6} M_{\odot}$ black holes in galactic nuclei has been estimated

\footnotetext{
${ }^{4}$ That is, $1 /\left(5 S_{h}\right) \equiv$ average over the sky of $1 /$ (spectral density). The factor 5 in this definition is to produce accord with the conventional notation for ground-based interferometers, where $S_{h}(f)$ denotes the spectral density for waves with optimal direction and polarization. In the case of LISA, at frequencies above about $0.01 \mathrm{~Hz}$, the beam pattern shows sharp frequency-dependent variations with direction due to the fact that the interferometer arms are acting as one-pass delay lines rather than optical cavities, and this produces a more complicated dependence of sensitivity on angle than for ground-based interferometers. As a result, $S_{h}$ (as we have defined it) is the spectral density for optimal direction and polarization only below about $0.01 \mathrm{~Hz}$, not above.
} 
TABLE II. Properties of the second-harmonic, transition-regime gravitational waves from a $\mu=10 M_{\odot}$ black hole spiraling into a $M=10^{6} M_{\odot}$ black hole (so $\eta=\mu / M=10^{-5}$ ) at $r=1 \mathrm{Gpc}$ distance. The values of $\dot{\mathcal{E}}_{\infty, 2}$ are from numerical solutions of the Teukolsky equation by Finn (first line of Table IV of Ref. [4]).

\begin{tabular}{|c|c|c|c|c|c|c|c|c|}
\hline$a$ & $f, \mathrm{~Hz}$ & $\frac{\Delta f}{f}$ & $\Delta t, \mathrm{sec}$ & $N_{\text {cyc }}$ & $\dot{\mathcal{E}}_{\infty, 2}$ & $h_{\mathrm{amp}}^{\mathrm{rms}}$ & & ms $10^{-22}$ \\
\hline-0.99 & 0.002496 & 0.033 & 9300 & 23 & 1.029 & 2.0 & & 1.2 \\
\hline-0.9 & 0.002601 & 0.033 & 8800 & 23 & 1.020 & 2.0 & & 1.2 \\
\hline-0.5 & 0.003188 & 0.037 & 7000 & 22 & 0.9734 & 2.3 & & 1.4 \\
\hline 0. & 0.004396 & 0.044 & 4800 & 21 & 0.8957 & 2.7 & & 1.6 \\
\hline 0.2 & 0.005167 & 0.047 & 4100 & 21 & 0.8535 & 2.9 & & 1.6 \\
\hline 0.5 & 0.007016 & 0.054 & 2900 & 21 & 0.7653 & 3.4 & & 1.6 \\
\hline 0.8 & 0.01123 & 0.062 & 1900 & 22 & 0.5914 & 4.1 & & 1.3 \\
\hline 0.9 & 0.01457 & 0.063 & 1700 & 24 & 0.4617 & 4.3 & & 1.1 \\
\hline 0.99 & 0.02354 & 0.051 & 1800 & 43 & 0.1656 & 3.6 & & 0.72 \\
\hline 0.999 & 0.02829 & 0.037 & 3400 & 96 & 0.06128 & 2.4 & & 0.58 \\
\hline
\end{tabular}

by Sigurdsson and Rees [2]; their "very conservative" result is $\sim$ one event per year out to $1 \mathrm{Gpc}$. The inspiraling holes are likely to be in rather eccentric, nonequatorial orbits [16], for which our analysis needs to be generalized. If, however, the orbit is circular and equatorial and the holes are at $1 \mathrm{Gpc}$ distance, then the above formulas give the numbers shown in Table II.

As shown in the table, the signal to noise for this source is of order unity. With some luck in the orientation of LISA, the orientation of the source, the distance to the source, and/or the holes' masses, a $S / N$ of a few might occur. Since the signal would already have been detected from the much stronger adiabatic inspiral waves, this signal strength could be enough to begin to explore the details of the transition from inspiral to plunge.

\section{CONCLUSIONS}

Our analysis of the transition regime has been confined to circular, equatorial orbits. This is a serious constraint, since there is strong reason to expect that most inspiraling bodies will be in orbits that are strongly noncircular and nonequatorial [16]. Our estimated signal-to-noise ratio, $S / N \sim 1$, for LISA's observations of the transition regime from a circular, equatorial orbit at the plausible distance $\sim 1$ Gpc suggests that for more realistic orbits the transition regime might be observable. This prospect makes it important to generalize our analysis to more realistic orbits.

Full analyses for equatorial, noncircular orbits and for nonequatorial, circular orbits can be carried out using techniques now in hand: the Teukolsky formalism, and computations of the orbital evolution based on the energy and angular momentum radiated down the hole and off to infinity (see, e.g., Ref. [5] and references therein). For nonequatorial, noncircular orbits, the analysis should also be possible with existing techniques - up to an unknown radiation-reactioninduced rate of evolution of the Carter constant. That unknown quantity could be left as a parameter in the analysis, to be determined when current research on gravitational radiation reaction $[17,18,6]$ has reached fruition.

When this paper was in near final form, we became aware of a similar analysis, by Buonanno and Damour [19], of the transition from inspiral to plunge. Whereas we treat the case of infinitesimal mass ratio $\eta \ll 1$ and finite black-hole spin $-1<a<+1$, Buananno and Damour treat finite $\eta(0<\eta$ $\leqslant 1 / 4$ ) and vanishing spins $a=0$. Both analyses give the same dimensionless equation of motion (3.22) for the transition regime.

\section{ACKNOWLEDGMENTS}

We thank James Anderson for helpful discussions, Theocharis Apostolatos for checking some details of our analysis, and Sam Finn for computing the numerical entries for $\dot{\mathcal{E}}$ and $\dot{\mathcal{E}}_{\infty, 2}$ in Tables I and II, on which many of our other numbers are based. This work was supported in part by NASA grant NAG5-6840, and in view of its potential applications to LIGO, also by NSF grant AST-9731698.
[1] For details of the LISA mission see http://lisa.jpl.nasa.gov/

[2] S. Sigurdsson and M. J. Rees, Mon. Not. R. Astron. Soc. 284, 318 (1997).

[3] S. Sigurdsson, Class. Quantum Grav. 14, 1425 (1997).

[4] L. S. Finn and K. S. Thorne, preceding paper, Phys. Rev. D 62, 124021 (2000).

[5] S. A. Hughes, Phys. Rev. D 61, 084004 (2000).
[6] L. Barack and A. Ori, Phys. Rev. D 61, 061502 (2000).

[7] C. W. Misner, K. S. Thorne, and J. A. Wheeler, Gravitation (Freeman, San Francisco, 1973).

[8] J. M. Bardeen, W. H. Press, and S. A. Teukolsky, Astrophys. J. 178, 347 (1972); J. M. Bardeen, in Black Holes, edited by C. DeWitt and B. S. DeWitt (Gordon and Breach, New York, 1973), p. 241. 
[9] Section 10.7 of Ya. B. Zel' dovich and I. D. Novikov, Relativistic Astrophysics, Vol. I, Stars and Relativity (U. Chicago Press, Chicago, 1971); also available as a Dover Reprint Volume.

[10] I. D. Novikov and K. S. Thorne, in Black Holes, edited by C. DeWitt and B. S. DeWitt (Ref. [8]), p. 343.

[11] L. E. Kidder, C. M. Will, and A. G. Wiseman, Phys. Rev. D 47, 3281 (1993).

[12] T. Damour, B. R. Iyer, and B. S. Sathyaprakash, Phys. Rev. D 57, 885 (1998).

[13] A. Buonanno and T. Damour, Phys. Rev. D 60, 023517 (1999).

[14] K. S. Thorne, in Three Hundred Years of Gravitation, edited by S. W. Hawking and W. Israel (Cambridge University Press, Cambridge, U.K., 1987), p. 330.

[15] LISA Mission Definition Team and LISA Science Study
Team, LISA: Laser Interferometer Space Antenna (March Press, Boulder, CO, 1999). The spectral density $S_{h}(f)$, as we have defined it, is related to the noise curve $h_{\text {noise }}(f)$ shown in the figure on page 6 by $S_{h}=3.156 \times 10^{7}\left(h_{\text {noise }}\right)^{2} / 125[\mathrm{P}$. Bender (private communication)]. Here $3.156 \times 10^{7}$ is the number of seconds in a year, and $125=(5 \sqrt{5})^{2}$ where the $\sqrt{5}$ comes from averaging the noise over the sky and the 5 from the fact that the figure in this document is for a signal to noise ratio of 5 .

[16] D. Hils and P. L. Bender, Astrophys. J. Lett. 445, L7 (1995); Y. Mino, M. Sasaki, M. Shibata, H. Tagoshi, and T. Tanaka, Suppl. Prog. Theor. Phys. 128, 1 (1998).

[17] Y. Mino, M. Sasaki, and T. Tanaka, Phys. Rev. D 55, 3457 (1997).

[18] T. C. Quinn and R. M. Wald, Phys. Rev. D 56, 3381 (1997).

[19] A. Buonanno and T. Damour, Phys. Rev. D 62, 064015 (2000). 